DOI: $10.24850 /$ j-tyca-2020-01-01

Artículos

\title{
Incertidumbre en la evaluación del impacto del cambio climático en dos cuencas mexicanas
}

\section{Uncertainty in the evaluation of climate change impacts over two Mexican catchments}

Juan Alberto Velázquez-Zapata ${ }^{1}$

Magali Troin ${ }^{2,3}$

${ }^{1}$ Consejo Nacional de Ciencia y Tecnología, El Colegio de San Luis, San Luis Potosí, México, javelazquezza@conacyt.mx, ORCID: 0000-00020208-9286

${ }^{2}$ Département du Génie de la Construction, École de Technologie Supérieure, Université du Québec, Montréal, Canada, magali.troin@etsmtl.ca, ORCID: 0000-0002-7811-9613

${ }^{3}$ HydroClimat, Toulon, France, magali.troin@hydroclimat.com

Autor para correspondencia: Juan Alberto Velázquez Zapata, javelazquezza@conacyt.mx

\section{Resumen}


Este estudio investiga la incertidumbre asociada con el modelo de circulación general (MCG) al escenario de emisión y a la variabilidad natural del clima en la estimación del impacto del cambio climático en dos cuencas mexicanas. Las cuencas seleccionadas tienen diferentes climas y regímenes hidrológicos. Las simulaciones climáticas de tres MCG bajo tres escenarios de emisión (B1, A1B y A2) se usan para alimentar el modelo hidrológico SWAT, de tipo físico y semidistribuido. Las simulaciones cubren 30 años en un periodo de referencia (19712000) y dos periodos de 20 años en el futuro (2046-2065 y 2081-2100). Los resultados muestran que los caudales altos son más propensos a ser influenciados por la incertidumbre asociada con el MCG; además, los indicadores hidrológicos tienen una sensibilidad menor al escenario de emisión para las cuencas de estudio. Por último, la incertidumbre vinculada con la variabilidad natural no debe pasarse por alto en los estudios de cambio climático, ya que hasta cierto punto y para caudales altos puede ser tan crítica como la incertidumbre ligada con MCG y el escenario de emisión.

Palabras clave: impacto del cambio climático, cadena de modelación, escurrimiento, incertidumbre, variabilidad natural, SWAT, México.

\section{Abstract}

This study explores the uncertainties associated with general circulation model (GCM), emission scenarios and natural climate variability into estimates of climate change impacts over two Mexican catchments. The two selected catchments have contrasted climate patterns and 
hydrological regimes. Climate ensemble simulations from three GCMs under three SRES scenarios (B1, A1B, and $A 2$ ) are considered to feed the physically-based semi-distributed SWAT hydrological model. The simulations cover the 30-year reference period (1971-2000) and two 20-year periods (2046-2065 and 2081-2100) in the future. For the set of hydrological indicators, the results show that the high flows are more prone to be influenced by the GCM uncertainty. A weak sensitivity of the hydrological indicators is observed to emission scenarios over the study catchments. We find that the uncertainty related to the natural climate variability should not be neglected in climate change impact studies, and appears, to some extent, to be as critical as the GCM and scenario uncertainties for high flows.

Keywords: Climate change impact, model chain, streamflow, uncertainty, natural variability, SWAT, Mexico.

Recibido: $31 / 08 / 2018$

Aceptado: 26/03/2019

\section{Introducción}




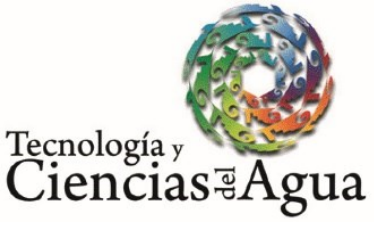

2020, Instituto Mexicano de Tecnología del Agua

Open Access bajo la licencia CC BY-NC-SA 4.0

(https://creativecommons.org/licenses/by-nc-sa/4.0/)

Las proyecciones de cambio climático para México y Centroamérica indican cambios en la precipitación y el incremento de la temperatura, lo que podría afectar la futura disponibilidad de los recursos hídricos en la región (IPCC, 2014). La Tabla 1 muestra algunos estudios sobre el impacto del cambio climático en los recursos hídricos en cuencas mexicanas. El uso de diferentes modelos de circulación general (MCG) permite estimar la incertidumbre relacionada con dichos modelos en la evaluación del impacto del cambio climático en los recursos hídricos. Por ejemplo, los trabajos de Velázquez-Zapata, Troin y Dávila-Ortiz (2017), y Molina-Navarro et al. (2016) evaluaron el impacto del cambio climático con 5 y 6 modelos, respectivamente. Por otro lado, el trabajo de Rivas, Güitrón y Montero (2011) combinó simulaciones climáticas de 23 modelos para obtener una simulación promedio. Sin embargo, el trabajo de Arnell y Gosling (2013) muestra que promediar simulaciones puede llevar a una pérdida de señal del cambio en la precipitación cuando existen modificaciones de diferente signo, además de que se podría subestimar la variabilidad espacial. Por otra parte, ninguno de los estudios mostrados en la Tabla 1 consideró la incertidumbre asociada con la variabilidad natural del clima (VNC). La VNC se estima al repetir un experimento de cambio climático con un MCG dado, pero cambiando ligeramente las condiciones iniciales (Braun, Caya, Frigon, \& Slivitzky, 2012). Respecto a esto, el trabajo de Deser, Knutti, Solomon y Phillips (2012) mostró que la fluctuación natural del clima contribuye de manera sustancial a la incertidumbre en las proyecciones de cambio climático en Norteamérica, sobre todo en latitudes altas. En contraste, los autores 
muestran que en las latitudes donde se encuentra México hay menos influencia de la variabilidad natural del clima.

Tabla 1. Estudios del impacto del cambio climático en recursos hídricos en cuencas mexicanas. La tabla muestra el número de Modelos de Circulación General (MCG) y de Modelos Climáticos Regionales (MCR) usados en el estudio.

\begin{tabular}{|c|c|c|c|c|c|c|}
\hline Referencia & Cuencas & $\begin{array}{c}\text { Indicado- } \\
\text { res }\end{array}$ & $\begin{array}{c}\text { Modelos } \\
\text { climáticos }\end{array}$ & $\begin{array}{c}\text { Escenario } \\
\text { de } \\
\text { emisión }\end{array}$ & Horizonte & $\begin{array}{c}\text { Variabilidad } \\
\text { natural }\end{array}$ \\
\hline $\begin{array}{c}\text { Mendoza, } \\
\text { Villanueva y } \\
\text { Adem } \\
\text { (1997) }\end{array}$ & $\begin{array}{c}\text { El territorio } \\
\text { nacional } \\
\text { dividido en } \\
12 \\
\text { regiones } \\
\text { hidroló- } \\
\text { gicas }\end{array}$ & $\begin{array}{c}\text { Índice de } \\
\text { vulnerabi- } \\
\text { lidad y } \\
\text { aridez (e.g., } \\
\text { disponibili- } \\
\text { dad de agua } \\
\text { y demanda) } \\
\text { Escurri- } \\
\text { miento } \\
\text { superficial } \\
\text { anual }\end{array}$ & 2 MCGs & No & 2050 & No \\
\hline $\begin{array}{c}\text { Gratiot et al. } \\
(2010)\end{array}$ & $\begin{array}{c}\text { Cointzio } \\
\left(650 \mathrm{~km}^{2}\right)\end{array}$ & $\begin{array}{c}\text { Índice de } \\
\text { aridez }\end{array}$ & $1 \mathrm{MCG}$ & A2 & $\begin{array}{c}\text { Décadas } \\
\text { centradas } \\
\text { en } 2030 \\
2060 \text { y } \\
2090\end{array}$ & No \\
\hline $\begin{array}{l}\text { Rivas, } \\
\text { Güitrón y } \\
\text { Montero } \\
\text { (2011) }\end{array}$ & $\begin{array}{l}\text { Lerma- } \\
\text { Chapala } \\
(54450 \\
\left.\mathrm{Km}^{2}\right)\end{array}$ & $\begin{array}{l}\text { Volumen de } \\
\text { caudal anual }\end{array}$ & $\begin{array}{l}\text { Promedio de } \\
23 \text { MCG }\end{array}$ & $A 1 B, A 2$ & $\begin{array}{c}2030 y \\
2050\end{array}$ & No \\
\hline $\begin{array}{c}\text { Maderey, } \\
\text { Jiménez y } \\
\text { Carrillo }\end{array}$ & $\begin{array}{l}\text { Lerma- } \\
\text { Chapala- } \\
\text { Santiago }\end{array}$ & $\begin{array}{l}\text { Disponibi- } \\
\text { lidad de } \\
\text { agua }\end{array}$ & $\begin{array}{l}2 \text { MCGs y un } \\
\text { modelo } \\
\text { termodiná- }\end{array}$ & $\begin{array}{c}\text { El } \\
\text { escenario } \\
\text { considera }\end{array}$ & $2025-2050$ & No \\
\hline
\end{tabular}


2020, Instituto Mexicano de Tecnología del Agua

Open Access bajo la licencia CC BY-NC-SA 4.0

Tecnología y

(https://creativecommons.org/licenses/by-nc-sa/4.0/)

\begin{tabular}{|c|c|c|c|c|c|c|}
\hline (2013) & $\begin{array}{c}(135836 \\
\left.\mathrm{km}^{2}\right) ; \\
\text { Balsas } \\
(117638 \\
\left.\mathrm{km}^{2}\right), y \\
\text { Pánuco (98 } \\
\left.302 \mathrm{~km}^{2}\right)\end{array}$ & superficial & mico & $\begin{array}{l}\text { el doble de } \\
\text { contenido } \\
\text { de } \mathrm{CO}_{2} \text { en } \\
\text { la } \\
\text { atmósfera }\end{array}$ & & \\
\hline $\begin{array}{c}\text { Tapia, } \\
\text { Minjarez, } \\
\text { Espinoza y } \\
\text { Minjarez } \\
(2014)\end{array}$ & $\begin{array}{c}\text { Río Yaqui } \\
(74054 \\
\left.\mathrm{km}^{2}\right)\end{array}$ & $\begin{array}{c}\text { Balance } \\
\text { hidrológico }\end{array}$ & $1 \mathrm{MCG}$ & $\mathrm{A} 1 \mathrm{~B}$ y $\mathrm{A} 2$ & 2010-2099 & No \\
\hline $\begin{array}{c}\text { Velázquez, } \\
\text { Troin y Caya } \\
\text { (2015) }\end{array}$ & $\begin{array}{c}\text { Río } \\
\text { Tampaón } \\
(23373 \\
\left.\mathrm{km}^{2}\right)\end{array}$ & $\begin{array}{c}\text { Indicadores } \\
\text { hidrológicos } \\
\text { promedio } \\
\text { mensuales }\end{array}$ & 1 MCG-MCR & $A 2$ & $2071-2100$ & No \\
\hline $\begin{array}{c}\text { Robles- } \\
\text { Morua, Che, } \\
\text { Mayer y } \\
\text { Vivoni } \\
(2015)\end{array}$ & $\begin{array}{c}\text { Río Sonora } \\
(20648 \\
\left.\mathrm{km}^{2}\right)\end{array}$ & $\begin{array}{l}\text { Caudal de } \\
\text { entrada de } \\
\text { embalses }\end{array}$ & 1 MCG-MRC & $A 2$ & $\begin{array}{c}2031- \\
2040\end{array}$ & No \\
\hline $\begin{array}{c}\text { Molina- } \\
\text { Navarro et } \\
\text { al. (2016) }\end{array}$ & $\begin{array}{c}\text { Río } \\
\text { Guadalupe } \\
(2380 \\
\left.\mathrm{km}^{2}\right)\end{array}$ & $\begin{array}{c}\text { Balance } \\
\text { hidrológico }\end{array}$ & 6 MCGs & $\mathrm{B} 1$ y $\mathrm{A} 2$ & $\begin{array}{c}2010-2039 \\
\text { y } 2070- \\
2099\end{array}$ & No \\
\hline $\begin{array}{c}\text { Velázquez- } \\
\text { Zapata et al. } \\
\text { (2017) }\end{array}$ & $\begin{array}{c}\text { Río } \\
\text { Tampaón } \\
(23373 \\
\left.\mathrm{km}^{2}\right)\end{array}$ & $\begin{array}{c}\text { Indicadores } \\
\text { hidrológicos } \\
\text { promedio } \\
\text { mensuales }\end{array}$ & 5 MCGs & $A 2$ & $2071-2100$ & No \\
\hline
\end{tabular}

La incertidumbre asociada con la VNC ha sido evaluada en algunos estudios del impacto del cambio climático en recursos hídricos en latitudes altas (p. ej., Velázquez et al., 2013). Sin embargo, la incertidumbre asociada con VNC en el impacto del cambio climático en 
indicadores hidrológicos no se ha evaluado en cuencas mexicanas (ver Tabla 1).

El objetivo de este estudio es la evaluación de las incertidumbres en la cadena de modelación del impacto del cambio climático en dos cuencas mexicanas con diferente régimen hidrológico. Para ello, se consideraron las simulaciones de tres MCGs bajo tres escenarios de emisión (A2, B1, y A1B) del Panel Intergubernamental del Cambio Climático (IPCC, 2010). Estas simulaciones climáticas se usaron como datos de entrada del modelo hidrológico físico semidistribuido SWAT (Soil and Water Assessment Tool) para un periodo de referencia (1971$2000)$ y dos periodos futuros (2046-2065 y 2081-2100). Por lo tanto, se consideraron tres fuentes de incertidumbre: MCGs, escenarios de emisión y variabilidad natural del clima.

En la Tabla 1 se puede observar que la mayoría de los estudios evalúan cambios en el balance hidrológico de la cuenca. En este estudio, la hidrología de la cuenca se evalúa con dos indicadores específicos: el caudal medio anual y el caudal alto.

El manuscrito está organizado de la siguiente manera: el área de estudio, las bases de datos meteorológicos observados y las simulaciones climáticas; el modelo hidrológico y el procedimiento de corrección de sesgo; los resultados de la evaluación del impacto del cambio climático en la hidrología de la cuenca y la estimación de la incertidumbre, y la al final se presentan conclusiones. 


\section{Diseño experimental}

\section{Cuencas de estudio}

La cuenca del río Tampaón (23 373 km²; IMTA, 2018) se localiza en la parte centro-este de México, principalmente en el estado de San Luis Potosí. El Tampaón ocupa una subcuenca del río Pánuco, el cual desemboca en el Golfo de México. Su relieve topográfico va de los $18 \mathrm{~m}$ a los 3500 m, con una elevación máxima en la Sierra Madre Oriental (Figura 1). Esta característica origina que la cuenca presente una variedad de regiones climáticas (Sedue, 1986; Peel, Finlayson, \& McMahon, 2007): el parteaguas más lejano se localiza en una región árida (BSk) y conforme fluye la corriente del oeste al este se encuentra en una región templada (Cwa). El río Valles, localizado en una región de clima tropical lluvioso (Aw y Am) se une al río Tampaón, proveyendo el volumen más importante a la corriente. 


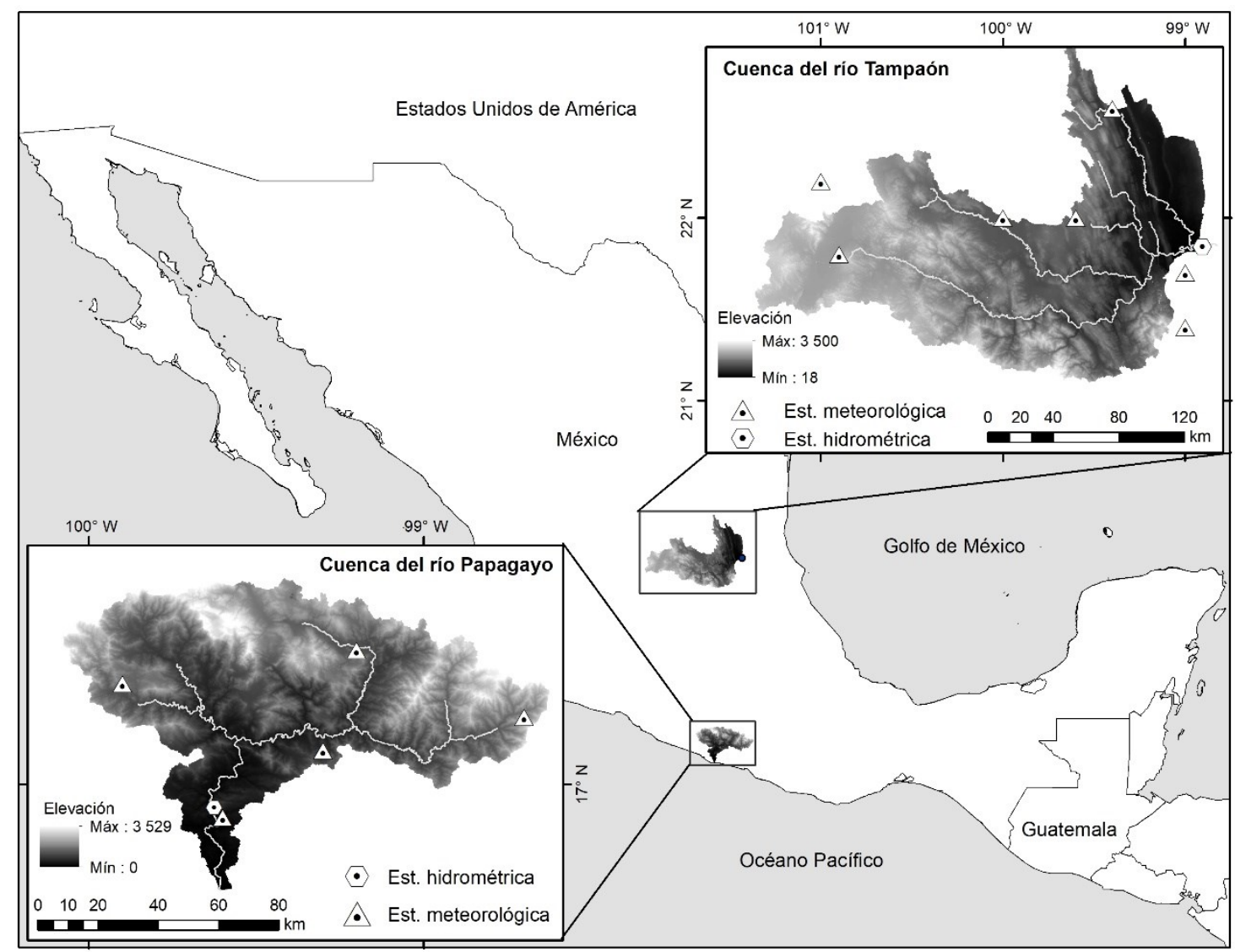

Figura 1. Localización de las cuencas de estudio.

La cuenca del río Papagayo se localiza por completo en el estado de Guerrero, al sur de México. Esta cuenca cubre un área de 7067 km² (IMTA, 2018), y presenta una elevación máxima de 3529 m (Figura 1). La cuenca del río Papagayo se localiza en una región climática Aw (tropical lluviosa), y la corriente principal fluye de la Sierra Madre del Sur hacia el océano Pacífico. 


\section{Datos meteorológicos observados}

Las series de temperatura máxima y mínima diaria, y de precipitación diaria se recuperaron de la base de datos CLICOM del servicio Meteorológico Nacional, vía el sitio web del Centro de Investigación Científica y de Educación Superior de Ensenada (CICESE, 2017). Se consideraron siete estaciones para la cuenca del río Tampaón y cinco para la cuenca del río Papagayo para el periodo 1971-2000 (Figura 1). La temperatura promedio anual es de $21.5^{\circ} \mathrm{C}$ y $24.1^{\circ} \mathrm{C}$ para las cuencas del río Tampaón y del río Papagayo, respectivamente. El mes más frío es enero y el más cálido es mayo (Figura 2). La estación de lluvias se extiende de mayo a octubre para ambas cuencas. La precipitación promedio anual es de $1080 \mathrm{~mm}$ y $1540 \mathrm{~mm}$ para las cuencas del río Tampaón y del río Papagayo, respectivamente. 

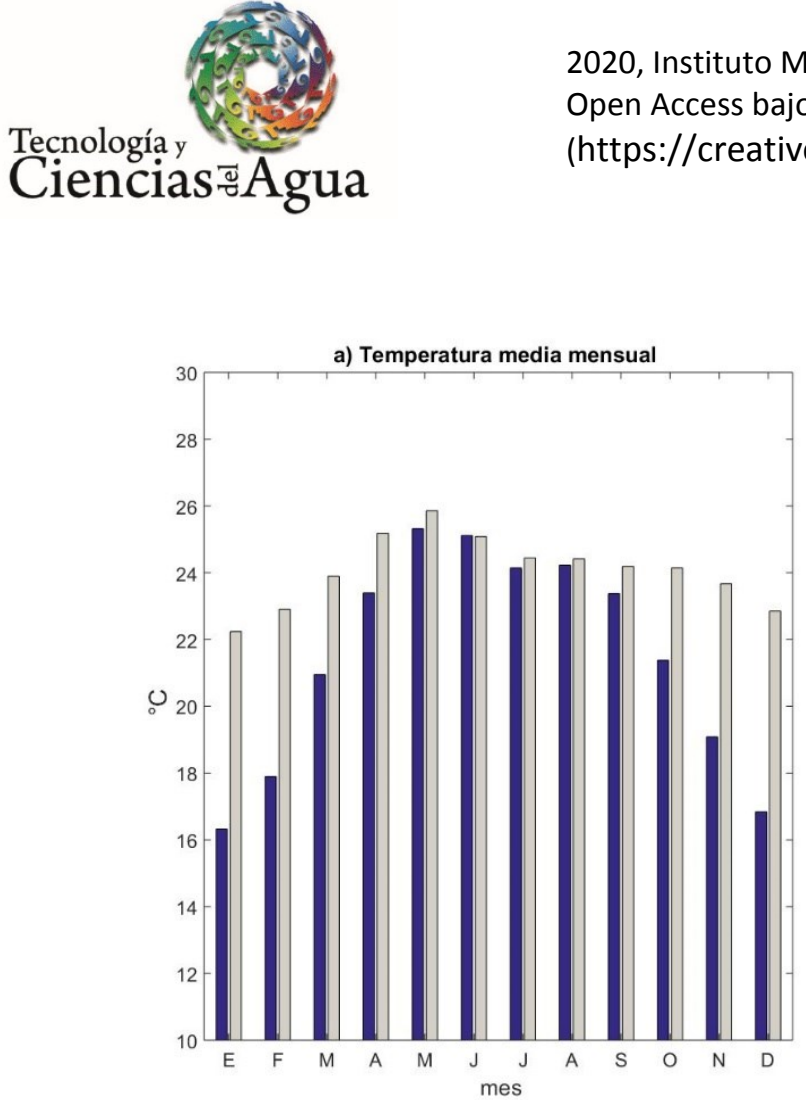

2020, Instituto Mexicano de Tecnología del Agua

Open Access bajo la licencia CC BY-NC-SA 4.0

(https://creativecommons.org/licenses/by-nc-sa/4.0/)

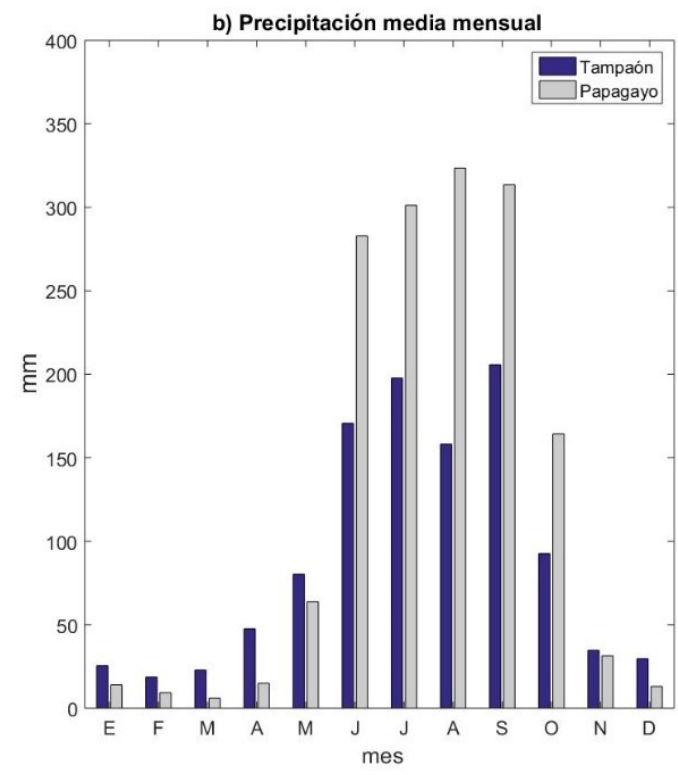

Figura 2. Temperatura y precipitación promedio mensual en las cuencas de estudio para el periodo 1971-2000.

Los datos de caudal se obtuvieron del Banco Nacional de Aguas Superficiales (BANDAS) (IMTA, 2018) para el periodo 1971-2000. La Figura 3 muestra el caudal promedio mensual para las dos cuencas. En la cuenca del río Tampaón se presentan dos picos de caudal en julio y septiembre, mientras que en la cuenca del río Papagayo hay un máximo en septiembre. En ambas cuencas, el pico de septiembre es de $450 \mathrm{~m}^{3}$ $\mathrm{s}^{-1}$ (47 $\mathrm{mm} \mathrm{mes}^{-1}$ y $164 \mathrm{~mm} \mathrm{mes}^{-1}$ para la cuenca del río Tampaón y la cuenca del río Papagayo, respectivamente). 

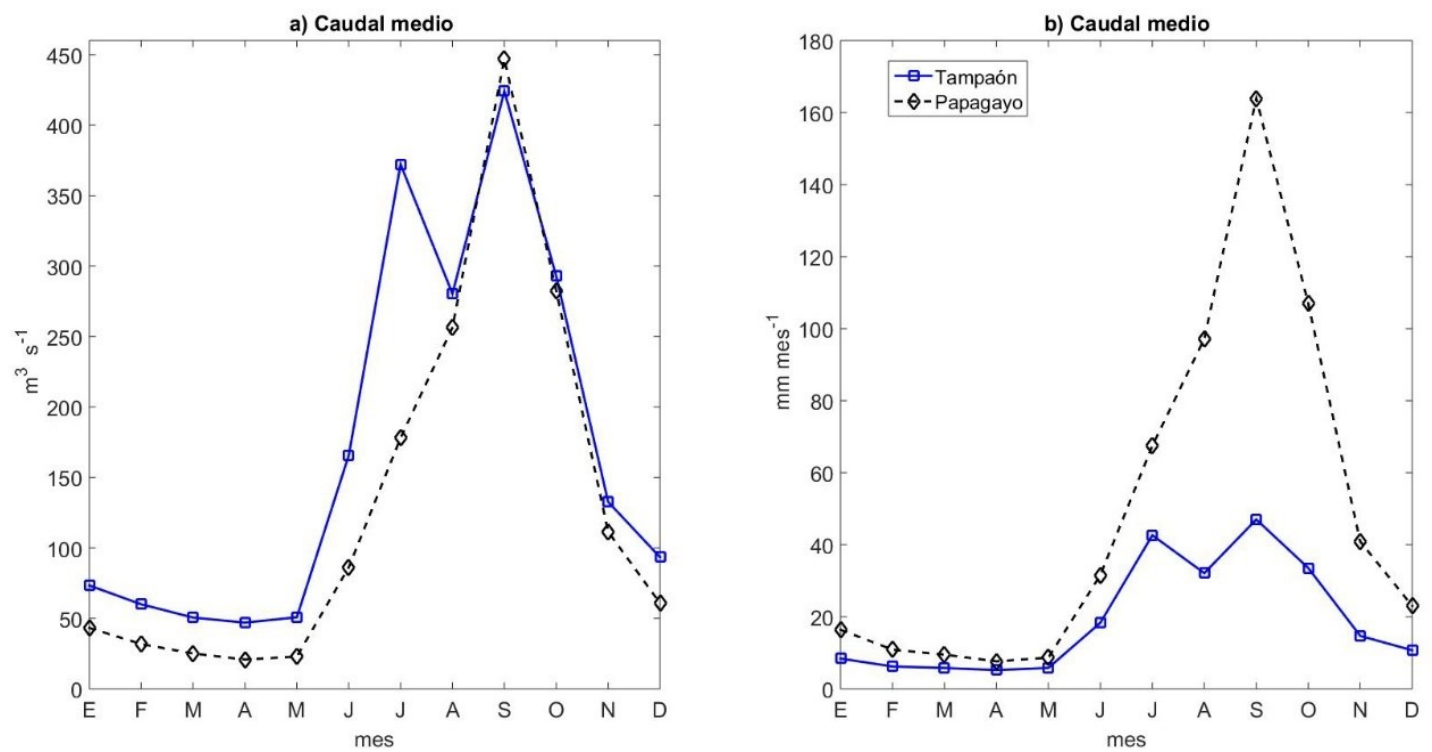

Figura 3. Caudal promedio mensual en las cuencas de estudio para el periodo 19712000.

\section{Los ensambles de simulaciones climáticas}

Las simulaciones climáticas se obtuvieron de tres MCGs del experimento multimodelo CMIP3 (Meehl et al., 2007) bajo tres escenarios de emisión (A1B, A2 y B1; ver Tabla 2). Tanto los MCGs como los escenarios de emisión se consideran igual de probables, por lo que las diferencias entre ellos al evaluar el impacto climático dan una idea de la incertidumbre de las futuras condiciones de los recursos hídricos en las 
cuencas. Los MCGs utilizados en este estudio se seleccionaron porque proveen un ensamble de simulaciones para cada escenario, lo que permite evaluar la incertidumbre relacionada con la variabilidad natural del clima.

Tabla 2. Modelos del experimento CMIP3 empleados en este estudio (ver Meehl et al., 2007, para la referencia de cada MCG).

\begin{tabular}{|c|c|c|c|}
\hline Instituto & $\begin{array}{c}\text { Identificador } \\
\text { CMIP3 }\end{array}$ & $\begin{array}{c}\text { Identificador } \\
\text { estudio }\end{array}$ & $\begin{array}{c}\text { Miem- } \\
\text { bros }\end{array}$ \\
\hline $\begin{array}{c}\text { Meteorological Institute of the University of } \\
\text { Bonn, }\end{array}$ & ECH & 3 \\
\hline $\begin{array}{r}\text { Canadian Centre for Climate Modelling \& } \\
\text { and Model and Data Group }\end{array}$ & ECHO-G & CGC & 5 \\
\hline CGCM3.1 & MRI- & & \\
\hline Meteorological Research Institute & CGCM2.3.2 & MRI & 5 \\
\hline
\end{tabular}

El uso directo de datos (precipitación y temperatura) de un MCG como entrada de un modelo hidrológico puede llevar a resultados poco realistas debido a los errores sistemáticos (sesgos) de las simulaciones climáticas (Jung, 2005). Por lo tanto, antes de usar estos datos es necesario ajustar la distribución de las simulaciones climáticas a la distribución de las observaciones a través de un modelo estadístico (Teutschbein, Wetterhall, \& Seibert, 2011). 
En el presente estudio, el método de procesamiento estadístico empleado es el propuesto por Mpelasoka y Chiew (2009), Ilamado daily translation (DT). En éste se obtiene una función de transferencia calculada con la diferencia (en percentiles) entre las distribuciones de frecuencias de las observaciones y simulaciones climáticas. Cuando se aplica el método DT a un ensamble de simulaciones, la función de transferencia se calcula con el promedio de las simulaciones y después cada miembro se ajusta de manera separada. Las variables meteorológicas se corrigen en el periodo de referencia con la Ecuación (1) y la Ecuación (2):

$$
\begin{aligned}
& T_{\operatorname{corr}(d)}^{r e f}=T_{\operatorname{sim}(d)}^{r e f}+\left(T_{o b s(m, q)}^{r e f}-T_{\operatorname{sim}(m, q)}^{r e f}\right) \\
& P_{\operatorname{corr}(d)}^{r e f}=P_{\operatorname{sim}(d)}^{r e f}\left(\frac{P_{o b s(m, q)}^{r e f}}{P_{\operatorname{sim}(m, q)}^{r e f}}\right)
\end{aligned}
$$

Donde $T_{\text {corr }}$ y $P_{\text {corr }}$ son la temperatura y precipitación corregidas en el periodo de referencia (ref) para un día dado $(d)$; los subíndices $m, q$, obs y sim indican mes, percentil, observaciones y simulaciones climáticas, respectivamente; en el periodo futuro (fut), las variables meteorológicas se corrigen con la Ecuación (3) y la Ecuación (4):

$T_{\text {corr }(d)}^{f u t}=T_{\operatorname{sim}(d)}^{f u t}+\left(T_{o b s(m, q)}^{r e f}-T_{\operatorname{sim}(m, q)}^{r e f}\right)$ 
$P_{\operatorname{corr}(d)}^{f u t}=P_{\operatorname{sim}(d)}^{f u t}\left(\frac{P_{o b s(m, q)}^{r e f}}{P_{\operatorname{sim}(m, q)}^{r e f}}\right)$

\section{Métodos}

La cadena de simulación hidroclimática consiste en un ensamble de simulaciones climáticas (corregidas) provenientes de tres MCG bajo tres escenarios de emisión ( $B 1, A 1 B$ y $A 2$ ) que se usan como datos de entrada de un modelo hidrológico. Los caudales simulados se analizan para estimar el impacto del cambio climático en diversos indicadores hidrológicos. Esta metodología permite evaluar la incertidumbre relacionada con el modelo climático, escenario de emisión y variabilidad natural del clima.

\section{El modelo hidrológico}


El modelo SWAT (Soil Water Assessment Tool) (Arnold, Srinivasan, Muttiah, \& Williams, 1998) es hidrológico de tipo físico semidistribuido. Se seleccionó debido a que ha sido usado ampliamente en estudios previos para evaluar el impacto del cambio climático en recursos hídricos (p. ej., Githui, Gitau, Mutua, Bauwens, 2009; Troin, Velázquez, Caya, \& Brissette, 2015). El modelo SWAT divide la cuenca en varias Unidades de Respuesta Hidrológica (URH), que toman en cuenta la variabilidad espacial (relieve, uso del suelo, tipo de suelo, etc.) de la cuenca. Para cada URH, el modelo SWAT simula el balance hidrológico de la cuenca en un paso de tiempo diario. El balance hidrológico se evalúa con la Ecuación (5):

$W_{t}=W_{o}+\sum_{i=1}^{t}\left(P_{i}-Q_{i s u f}-E T_{i}-w_{i}-Q_{i g w}\right)$

Donde $W t$ es la humedad del suelo en un tiempo $t ; W_{o}$, la humedad inicial del suelo. Para un día dado $i, P_{i}$ es la precipitación; $Q_{\text {isurf, }}$ el escurrimiento superficial; $E T_{i}$, la evapotranspiración; $w_{i}$, el agua percolada a través del perfil del suelo, y $Q_{i g w}$ es el flujo subterráneo. Todos los términos se expresan en $\mathrm{mm}$ de agua. Las referencias detalladas del modelo se dan en Neitsch, Arnold, Kiniry, Williams y King (2002).

El modelo SWAT se calibró en el periodo 1971-1985 y se validó en el periodo 1986-2000 para ambas cuencas. La eficacia del modelo se evaluó con el coeficiente de Nash-Sutcliffe (NS). Los resultados muestran que SWAT simula de forma adecuada el caudal en los periodos 
de calibración y validación, con valores de NS de 0.91 y 0.85, respectivamente, para la cuenca del río Tampaón, y valores de 0.86 y 0.84 en calibración y validación para la cuenca del río Papagayo.

\section{Indicadores hidrológicos}

Se seleccionaron dos indicadores hidrológicos para evaluar el impacto del cambio climático en hidrología de las cuencas:

1. El caudal medio diario (OMF).

2. El caudal con periodo de retorno de dos años (HF2). Para calcularlo, se supone que la serie sigue la función de distribución de probabilidad log Pearson III (p. ej., Muerth et al., 2013; Troin et al., 2015).

Los MCGs usados en este estudio proveen un ensamble de simulaciones climáticas que se usan para la evaluación de incertidumbre de la VNC. Para ello, los cambios relativos en los indicadores hidrológicos se evalúan con permutaciones, tal como propone el trabajo de Velázquez et al. (2013). Este método asume que cada miembro del ensamble (en ambos periodos) es una representación independiente del clima. Por lo tanto, la permutación permite comparar el clima futuro de un miembro dado con el clima presente de todos los miembros del ensamble. 
Las diferencias relativas en los indicadores hidrológicos $\left(\Delta_{i j}\right)$ se calculan con la Ecuación (6):

$\Delta_{i j}=\frac{I_{i}^{f u t}-I_{j}^{r e f}}{I_{j}^{r e f}}$

En la Ecuación (6), los subíndices $i$ y $j$ representan las simulaciones climáticas usadas para evaluar los indicadores hidrológicos. Por lo tanto, con CGC y MRI se obtienen 25 valores de cambio relativo mientras que con $\mathrm{ECH}$ se obtienen nueve. El diagrama de caja de los cambios relativos (como se muestra en la Figura 4) da una estimación de la incertidumbre relacionada con la variabilidad natural del clima. 
Periodo de referencia

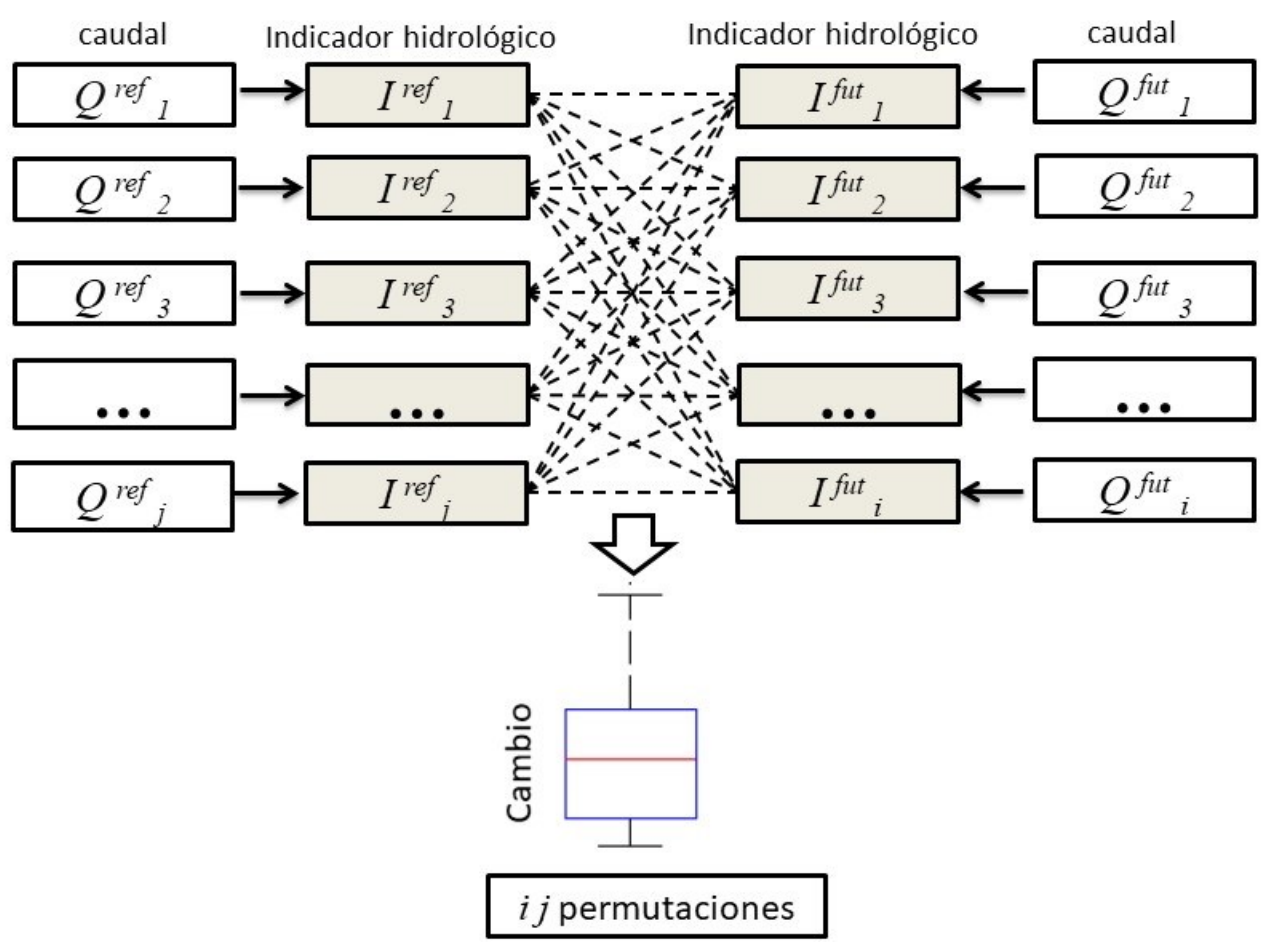

Figura 4. Diagrama del proceso de permutación.

El impacto del cambio climático en los indicadores hidrológicos $(\Delta I)$ se expresa como la diferencia entre los indicadores evaluados en el periodo $\left(I^{\text {ref }}\right)$ de referencia y el periodo futuro $\left(I^{\text {fut }}\right)$. 


\section{Resultados}

\section{Cambios en temperatura y precipitación en el periodo futuro}

Los cambios en precipitación y temperatura se estiman al analizar las diferencias en las simulaciones climáticas (corregidas) entre los periodos futuros (2046-2065 y 2081-2100) y el periodo de referencia (19712000). La Figura 5 y Figura 6 muestran las señales de cambio climático para la temperatura media anual y la precipitación media anual para las cuencas del río Tampaón y del río Papagayo, respectivamente. Los resultados indican que, en ambos periodos futuros, se estima un aumento de la temperatura. Sin embargo, los MCGs proyectan diferentes valores de incremento para un escenario de emisión dado. Los tres escenarios de emisión utilizados en este estudio ( $A 1 B, B 1$ y A2) consideran diferentes hipótesis sobre la población futura en relación con la manera de producir y utilizar energía (IPCC, 2010). Como es de esperar, el mayor cambio de temperatura es estimado para el horizonte 2081-2100 bajo el escenario A2, seguido de los escenarios A1B y B1. El incremento de la temperatura también varía dependiendo del MCG: los 
modelos ECH y CGC por lo general presentan los menores y mayores incrementos de temperatura de manera respectiva.


Figura 5. Señal de cambio climático en la cuenca del río Tampaón, estimada con los tres MCGs para los horizontes 2046-2065 (paneles superiores) y 2081-2100 (paneles inferiores) bajo los escenarios B1 (a y d), A1B (b y e) y A2 (c y f). 
Tecnología y

\section{Ciencias $₫$ Agua}
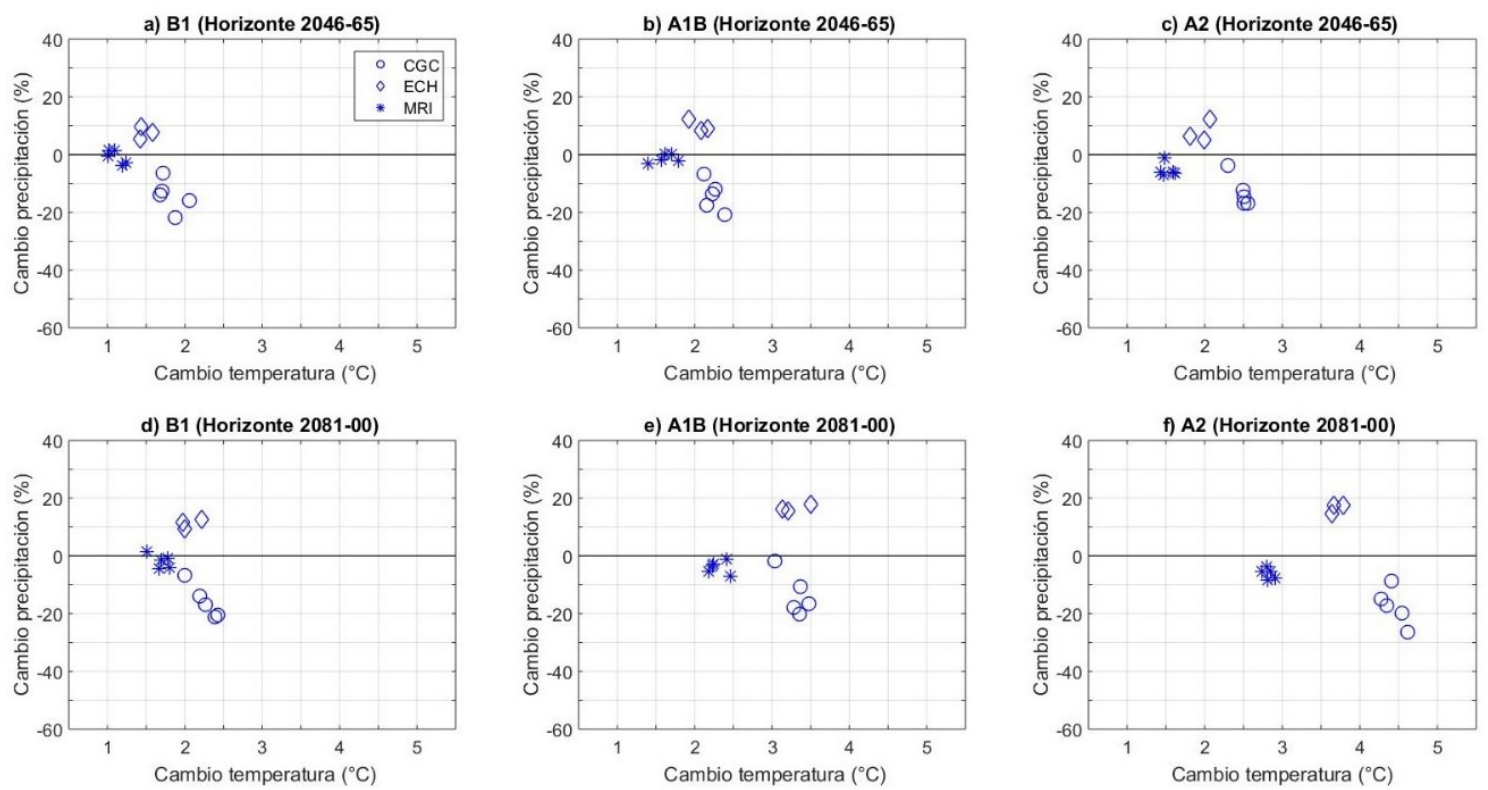

Figura 6. Similar a la Figura 5 para la cuenca del río Papagayo.

Los resultados son más variados para la precipitación. Así, en la Figura 5 y Figura 6 se puede ver que, en las dos cuencas, el modelo CGC estima un decremento en la precipitación, mientras que ECH evalúa un incremento. Por otro lado, MRI proyecta pocos cambios en el futuro para ambas cuencas.

Además, la Figura 5 y Figura 6 muestran que los valores de cambio de la temperatura y precipitación para el periodo 2081-2100 están más extendidos que en el horizonte 2046-2065, en especial para el escenario A2. Este resultado destaca la forma en que la incertidumbre varía según se considera un escenario de emisión y un horizonte de predicción dado. Asimismo, se puede ver que los cambios relativos pueden estar superpuestos entre los MCGs (p. ej., horizonte 2046-2065 
bajo el escenario B1; Figura 5a), lo que muestra que en este horizonte y con este escenario, la incertidumbre relacionada con la VNC es similar a la incertidumbre vinculada con el MCG. En contraste, la incertidumbre asociada con la VNC se vuelve menos importante en el horizonte 20812100 (Figura 5f). Tal resultado está de acuerdo con el trabajo de Hawkins y Sutton (2009), el cual analiza diferentes fuentes de incertidumbre en la predicción de la temperatura. Los autores de este trabajo mostraron que el peso específico que tiene cada fuente de incertidumbre varía con el escenario de emisión y con el horizonte de predicción. Por lo tanto, las fuentes dominantes de incertidumbre (con mayor peso) son el modelo climático y el escenario de emisión cuando se consideran los horizontes de predicción largos.

\section{Incertidumbre asociada con la variabilidad natural del clima en los indicadores hidrológicos}

La Figura 7 y Figura 8 muestran, para los indicadores hidrológicos seleccionados, los cambios relativos entre el periodo de referencia (1971-2000) y el periodo futuro (2046-2065) evaluados con la Ecuación (6), para la cuenca del río Tampaón y la cuenca del río Papagayo, respectivamente. 



Figura 7. Cambios relativos (evaluados con la Ecuación (6)) para el caudal medio (OMF, paneles superiores) y el caudal alto con periodo de retorno de dos años (HF2, paneles inferiores) entre el periodo de referencia (1971-00) y el periodo futuro (horizonte 2046-65) para la cuenca del río Tampaón. Los diagramas de caja muestran la mediana, y los percentiles 25 y 75. 
2020, Instituto Mexicano de Tecnología del Agua

Open Access bajo la licencia CC BY-NC-SA 4.0

Tecnología y

(https://creativecommons.org/licenses/by-nc-sa/4.0/)
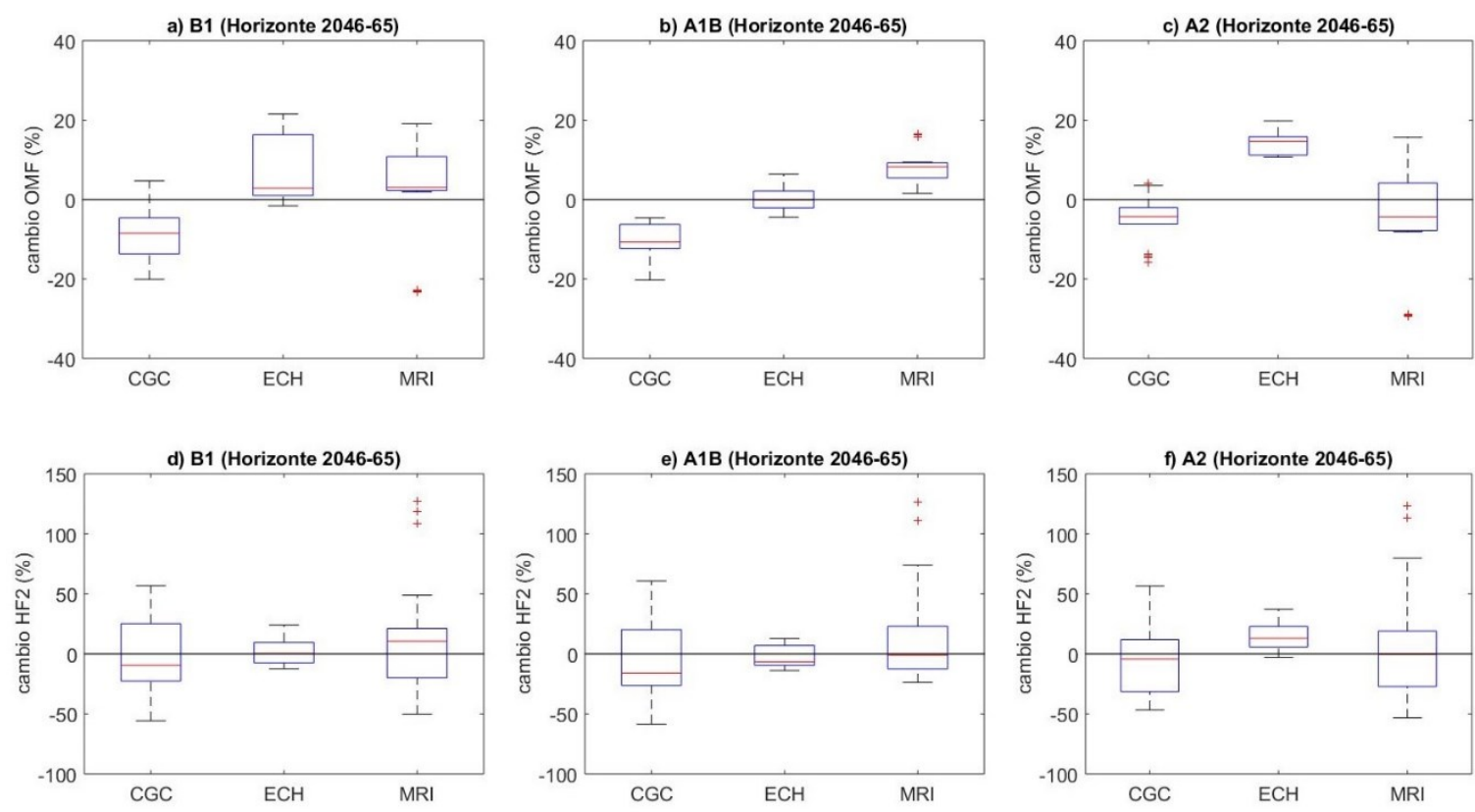

Figura 8. Similar a la Figura 7, pero para la cuenca del río Papagayo.

A partir de estas figuras se puede observar que por lo general GCG conduce a valores negativos de OMF. Sin embargo, ECH y MRI producen valores tanto positivos como negativos para este indicador. Por ejemplo, para la cuenca del río Papagayo bajo el escenario A1B (Figura $8 \mathrm{~b}$ ), los valores de OMF para $\mathrm{ECH}$ varían entre $-4.5 \%$ y $+6.3 \%$, con un valor mediano de $-0.1 \%$. Se puede notar que en algunos casos el rango de valores de cambios en OMF obtenidos con un MCG es cubierto por los cambios obtenidos con otro modelo. Por ejemplo, en la cuenca del río Tampaón (escenario A1B, Figura 7), los valores de cambios relativos para OMF obtenidos con MRI varían entre $-5.5 \%$ y $17 \%$, y cubre los valores de cambios relativos obtenidos con $\mathrm{ECH}$ (entre 7 y $9 \%$, aunque el valor mediano de cambio en OMF para los dos modelos es muy cercano ( $8 \%)$. Esta situación es más común para el indicador de 
caudal alto (Figura 7d, Figura 7e y Figura 7f), lo cual indica que la señal de cambio climático en HF2 obtenida con los diferentes MCG puede ser similar debido a la incertidumbre relacionada con la VNC.

El test de suma de rangos de Wilcoxon (Wilcoxon, 1945) fue hecho para evaluar si dos muestras de cambios relativos, obtenidas de distintos MCG, son independientes o no. En las tablas siguientes se presentan los resultados del test de Wilcoxon que muestran el rechazo (o no) de la hipótesis nula, la cual asume que dos series son muestras independientes de distribuciones continuas idénticas con medianas iguales. La Tabla 3 y Tabla 4 comparan (en pares), para un MCG dado, las series de cambio en los indicadores para los tres escenarios de emisión ( $B 1, A 1 B$ y $A 2$ ) para las cuencas del río Tampaón y del río Papagayo, respectivamente. De manera similar, la Tabla 5 y Tabla 6 comparan (en pares), las series de cambio en los indicadores para los tres MCG (CGC, ECH y MRI) para las cuencas del río Tampaón y del río Papagayo, respectivamente. Las áreas grises en la Tabla 3, Tabla 4, Tabla 5 y Tabla 6 indican que la hipótesis nula no es rechazada, esto es, que los pares de series tienen una señal de cambio climático similar debido a la variabilidad natural del clima.

Tabla 3. Resultados del test de Wilcoxon ( $p$-values) en la comparación de pares de escenarios para la cuenca del río Tampaón. Las áreas grises indican que la hipótesis nula no fue rechazada a un nivel de significancia de $5 \%$.

\begin{tabular}{|l|l|l|l|}
\hline & CGC & ECH & MRI \\
\hline
\end{tabular}


Tecnología y

Ciencias
2020, Instituto Mexicano de Tecnología del Agua

Open Access bajo la licencia CC BY-NC-SA 4.0

(https://creativecommons.org/licenses/by-nc-sa/4.0/)

\begin{tabular}{|c|c|c|c|c|c|c|c|c|c|c|}
\hline Ind. & Hor. & $\begin{array}{l}\text { B1- } \\
\text { A1B }\end{array}$ & $\mathrm{B} 1-\mathrm{A} 2$ & A1B-A2 & $\begin{array}{l}\text { B1- } \\
\text { A1B }\end{array}$ & $B 1-A 2$ & A1B-A2 & $\begin{array}{l}\text { B1- } \\
\text { A1B }\end{array}$ & $B 1-A 2$ & A1B-A2 \\
\hline \multirow[t]{2}{*}{ OMF } & $\begin{array}{l}2046- \\
2065\end{array}$ & 0.712 & 0.030 & 0.088 & 0.001 & 0.605 & $<0.001$ & $<0.001$ & $<0.001$ & 0.229 \\
\hline & $\begin{array}{l}2081- \\
2100\end{array}$ & $<0.001$ & $<0.001$ & 0.002 & $<0.001$ & $<0.001$ & 0.114 & 0.063 & 0.003 & 0.081 \\
\hline \multirow[t]{2}{*}{ HF2 } & $\begin{array}{l}2046- \\
2065\end{array}$ & 0.954 & 0.222 & 0.174 & 0.796 & 0.436 & 0.863 & 0.125 & 0.222 & 0.907 \\
\hline & $\begin{array}{l}2081- \\
2100\end{array}$ & 0.383 & 0.112 & 0.269 & 0.436 & 0.436 & 0.436 & 0.712 & 0.628 & 0.756 \\
\hline
\end{tabular}

Tabla 4. Similar a la Tabla 3, pero para la cuenca del río Papagayo.

\begin{tabular}{|c|c|c|c|c|c|c|c|c|c|c|}
\hline \multirow{2}{*}{ Ind. } & \multirow[b]{2}{*}{ Hor. } & \multicolumn{3}{|c|}{ CGC } & \multicolumn{3}{|c|}{ ECH } & \multicolumn{3}{|c|}{ MRI } \\
\hline & & $\begin{array}{l}\text { B1- } \\
\text { A1B }\end{array}$ & $\mathrm{B} 1-\mathrm{A} 2$ & A1B-A2 & $\begin{array}{c}\text { B1- } \\
\text { A1B }\end{array}$ & $\mathrm{B} 1-\mathrm{A} 2$ & $A 1 B-A 2$ & $\begin{array}{l}\text { B1- } \\
\text { A1B }\end{array}$ & $\mathrm{B} 1-\mathrm{A} 2$ & A1B-A2 \\
\hline \multirow[t]{2}{*}{ OMF } & $\begin{array}{l}2046- \\
2065\end{array}$ & 0.4263 & 0.0436 & $<0.001$ & 0.077 & 0.1359 & $<0.001$ & 0.4263 & 0.0914 & $<0.001$ \\
\hline & $\begin{array}{l}2081- \\
2100\end{array}$ & 0.415 & 0.017 & 0.2443 & $<0.001$ & 0.014 & 0.6048 & 0.4492 & 0.0298 & 0.229 \\
\hline \multirow[t]{2}{*}{ HF2 } & $\begin{array}{l}2046- \\
2065\end{array}$ & 0.6554 & 0.8461 & 0.6414 & 0.3401 & 0.0625 & 0.004 & 0.8766 & 0.4492 & 0.4263 \\
\hline & \begin{tabular}{|l|}
$2081-$ \\
2100
\end{tabular} & 0.9227 & 0.393 & 0.3825 & 0.3401 & 0.3865 & 0.9314 & 0.6695 & 0.6004 & $>0.99$ \\
\hline
\end{tabular}


Tabla 5. Resultados del test de Wilcoxon ( $p$-values) en la comparación de pares de MCG para la cuenca del río Tampaón. Las áreas grises indican que la hipótesis nula no fue rechazada a un nivel de significancia de $5 \%$.

\begin{tabular}{|c|c|c|c|c|c|c|c|c|c|c|}
\hline & \multicolumn{4}{|c|}{ CGC-ECH } & \multicolumn{3}{|c|}{ CGC-MRI } & \multicolumn{3}{|c|}{ ECH-MRI } \\
\hline Ind. & Hor. & B1 & $A 1 B$ & A2 & B1 & $A 1 B$ & $\mathrm{~A} 2$ & B1 & $A 1 B$ & $\mathrm{~A} 2$ \\
\hline \multirow{2}{*}{ OMF } & $\begin{array}{l}2046- \\
2065\end{array}$ & $<0.001$ & $<0.001$ & $<0.001$ & $<0.001$ & $<0.001$ & $<0.001$ & 0.258 & 0.310 & 0.118 \\
\hline & $\begin{array}{l}2081- \\
2100\end{array}$ & $<0.001$ & $<0.001$ & $<0.001$ & $<0.001$ & $<0.001$ & $<0.001$ & $<0.001$ & 0.532 & 0.009 \\
\hline \multirow{2}{*}{ HF2 } & $\begin{array}{l}2046- \\
2065\end{array}$ & 0.086 & 0.029 & 0.019 & 0.214 & 0.015 & 0.001 & 0.292 & 0.458 & 0.435 \\
\hline & $\begin{array}{l}2081- \\
2100\end{array}$ & 0.001 & 0.001 & $<0.001$ & $<0.001$ & $<0.001$ & $<0.001$ & 0.226 & 0.086 & 0.017 \\
\hline
\end{tabular}

Tabla 6. Similar a la Tabla 5, pero para la cuenca del río Papagayo.

\begin{tabular}{|c|c|c|c|c|c|c|c|c|c|c|}
\hline & \multicolumn{5}{|c|}{ CGC-ECH } & \multicolumn{3}{c|}{ CGC-MRI } & \multicolumn{3}{c|}{ ECH-MRI } \\
\hline Ind. & Hor. & B1 & A1B & A2 & B1 & A1B & A2 & B1 & A1B & A2 \\
\hline \multirow{2}{*}{ OMF } & $\begin{array}{l}2046- \\
2065\end{array}$ & $<0.001$ & $<0.001$ & $<0.001$ & 0.005 & $<0.001$ & 0.756 & 0.969 & $<0.001$ & 0.002 \\
\cline { 2 - 12 } & $\begin{array}{l}2081- \\
2100\end{array}$ & $<0.001$ & $<0.001$ & $<0.001$ & 0.002 & 0.074 & 0.006 & $<0.001$ & $<0.001$ & $<0.001$ \\
\hline \multirow{2}{*}{ HF2 } & $\begin{array}{l}2046- \\
2065\end{array}$ & 0.369 & 0.274 & 0.042 & 0.304 & 0.027 & 0.641 & 0.585 & 0.482 & 0.160 \\
\cline { 2 - 12 } & $2081-$ & 0.032 & 0.009 & 0.067 & 0.187 & 0.214 & 0.587 & 0.585 & 0.056 & 0.051 \\
\hline
\end{tabular}


Así, se puede ver en la Tabla 3 y Tabla 4 que cuando se comparan los escenarios de emisión, la hipótesis nula no es rechazada en todos los casos de HF2. Los resultados para OMF son mixtos, por ejemplo, para el río Tampaón, en el periodo 2081-2100, la hipótesis nula fue rechazada para todos los casos de CGC, y en algunos casos de ECH y MRI (Tabla $3)$.

Por otro lado, cuando los pares de series de cambios son comparados por MCGs (Tabla 5 y Tabla 6), los resultados muestran un rechazo general de la hipótesis nula para el indicador OMF. La excepción se da para el río Tampaón en el horizonte 2046-2065, en la que la hipótesis nula no es rechazada para el par ECH-MRI en ningún escenario. En el caso de OMF para el río Papagayo (Tabla 6), la comparación en las series de cambios muestra que la hipótesis nula no fue rechazada para tres casos aislados. Sin embargo, los resultados son diferentes en el caso del indicador de caudal alto (HF2): para el río Tampaón (Tabla 5), la hipótesis nula no es rechazada en la mayoría de los casos cuando los pares ECH-MRI son evaluados. De manera similar para la cuenca del río Papagayo (Tabla 6) no hay rechazo de la hipótesis nula en la mayoría de los pares CGC-MRI y en todos los pares ECH-MRI.

Los resultados anteriores muestran que existe una incertidumbre importante en la evaluación del impacto del cambio climático del caudal alto (HF2), y que ésta se relaciona más con la VNC que con la elección del MCG o el escenario de emisión. Por otro lado, se puede observar que 
la incertidumbre asociada con la VNC es menos importante para el caudal medio (OMF) que para el caudal alto.

\section{Conclusión}

El objetivo de este trabajo fue evaluar el impacto del cambio climático en dos cuencas mexicanas con diferente régimen hidrológico, considerando tres tipos de incertidumbre: el modelo climático (MCG), el escenario de emisión y la variabilidad natural del clima (VNC).

En lo que respecta a los cambios proyectados en las variables meteorológicas, el análisis mostró que en las dos cuencas hay una gran variabilidad en la precipitación, dado que los MCGs seleccionados estiman tanto incremento como decremento en el futuro. Además, la incertidumbre asociada con la VNC es una fuente importante de incertidumbre, dependiendo del escenario de emisión considerado.

Estudios previos muestran que la fuente más importante de incertidumbre en la evaluación del impacto del cambio climático en los recursos hídricos es la elección del MCG (p. ej., Graham, Hagemann, Jaun, \& Beniston, 2007). Los resultados del presente estudio muestran que la incertidumbre asociada con el modelo climático es un factor 
determinante, sin embargo, la incertidumbre ligada con la VNC no puede ser subestimada. Así, los resultados muestran que la incertidumbre relacionada con la VNC es crítica en el caso de caudales altos, y hasta cierto punto comparable con la incertidumbre asociada con MCG y el escenario de emisión.

El trabajo en el futuro deberá incluir más cuencas de estudio, con el fin de explorar los diversos climas en el territorio mexicano. Además, deberán evaluarse otras fuentes de incertidumbre, como la elección del método de corrección de sesgo y el modelo hidrológico. Por último, este trabajo se basó en simulaciones climáticas del experimento CMIP3, por lo que se deberán también considerar simulaciones del experimento CMIP5.

\section{Agradecimientos}

Los autores agradecen a los grupos de investigación del PCMDI (Program for Climate Model Diagnosis and Intercomparison) y del WGCM (WCRP's Working Group on Coupled Modelling), que hicieron disponibles las simulaciones climáticas.

\section{Referencias}

Arnell, N. G., \& Gosling, S. N. (2013). The impacts of climate change on river flow regimes at the global scale. Journal of Hydrology, 486, 351-364. 
Arnold, J. G., Srinivasan, R., Muttiah, R. S., \& Williams, J. R. (1998). Large area hydrologic modeling and assessment part I: Model development 1. JAWRA Journal of the American Water Resources Association, 34(1), 73-89.

Braun, M., Caya, D., Frigon, A., \& Slivitzky, M. (2012). Internal variability of Canadian RCM's hydrological variables at the basin scale in Quebec and Labrador. Journal of Hydrometeorology, 13, 443-462.

CICESE, Centro de Investigación Científica y de Educación Superior de Ensenada. (2017). Base de datos climatológica nacional. Ensenada, México: Centro de Investigación Científica y de Educación Superior de Ensenada. Recuperado de http://clicommex.cicese. $\mathrm{mx}$

Deser, C., Knutti, R., Solomon, S., \& Phillips, A. S. (2012). Communication of the role of natural variability in future North American climate. Nature Climate Change, 2(11), 775-779.

Githui, F., Gitau, W., Mutua, F., \& Bauwens, W. (2009). Climate change impact on SWAT simulated streamflow in western Kenya. International Journal of Climatology, 29, 1823-1834.

Graham, L. P., Hagemann, S., Jaun, S., \& Beniston, M. (2007). On interpreting hydrological change from regional climate models. Climatic Change, 81, 97-122.

Gratiot, N., Duvert, C., Collet, L., Vinson, D., Némery, J., \& SáenzRomero, C. (2010). Increase in surface runoff in the central 
mountains of Mexico: Lessons from the past and predictive scenario for the next century. Hydrology and Earth System Sciences, 14, 291-300. DOI: 10.5194/hess-14-291-2010

Hawkins, E., \& Sutton, R. (2009). The potential to narrow uncertainty in regional climate predictions. Bulletin of the American Meteorological Society, 90, 1095-1107.

IMTA, Instituto Mexicano de Tecnología del Agua. (2018). Banco Nacional de Datos de Aguas Superficiales (BANDAS). Jiutepec, México: Instituto Mexicano de Tecnología del Agua. Recuperado de https://www.imta.gob.mx/bandas

IPCC, Intergovernmental Panel on Climate Change. (2010). Emissions Scenarios: IPCC Special Report. Cambridge, UK: Cambridge University Press.

IPCC, Intergovernmental Panel on Climate Change. (2014). Contribution of Working Group II to the Fifth Assessment Report of the Intergovernmental Panel on Climate Change: Impacts, adaptation, and vulnerability. Cambridge, UK, \& New York, USA: Cambridge University Press.

Jung, T. (2005). Systematic errors of the atmospheric circulation in the ECMWF forecasting system. Quarterly Journal of the Royal Meteorological Society, 131, 1045-1073. DOI:10.1256/qj.04.93

Maderey, L. E., Jiménez, A., \& Carrillo, J. J. (2013). Global climate change and its effect on hydrological resources of Mexico's central 
region. Analele stiintifice ale Universitatii "Alexandru Ioan Cuza" din Iasi-seria Geografie, 59(1), 59-78.

Meehl, G. A., Covey, C., Taylor, K. E., Delworth, T., Stouffer, R. J., Latif, M., McAvaney, B., \& Mitchell, J. F. B. (2007). THE WCRP CMIP3 Multimodel dataset: A new era. Climate Change Research. Bulletin of the American Meteorological Society, 88, 1383-1394.

Mendoza, V. M., Villanueva, E. E., \& Adem, J. (1997). Vulnerability of basins and watersheds in Mexico to global change. Climate Research, 9, 139-145.

Molina-Navarro, E., Hallack-Alegría, M., Martínez-Pérez, S., RamírezHernández, J., Mungaray-Moctezuma, A., \& Sastre-Merlín, A. (2016). Hydrological modeling and climate change impacts in an agricultural semiarid region. Case study: Guadalupe River Basin, Mexico. Agricultural Water Management, 175, 29-42.

Mpelasoka, F. S., \& Chiew, F. H. S. (2009). Influence of rainfall scenario construction methods on runoff projections. Journal of Hydrometeorology, 10, 1168-1183.

Muerth, M. J., Gauvin St-Denis, B., Ricard, S., Velázquez, J. A., Schmid J., Minville, M., Caya, D., Chaumont, D., Ludwig, R., \& Turcotte, R. (2013). On the need for bias correction in regional climate scenarios to assess climate change impacts on river runoff. Hydrology and Earth System Sciences, 17, 1189-1204. 
Neitsch, S. L., Arnold, J. G., Kiniry, J. R., Williams, J. R., \& King, K. W. (2002). Soil and water assessment tool theoretical documentation version 2000. Texas, USA: Texas Water Resources Institutes.

Peel, M. C., Finlayson, B. L., \& McMahon, T. A. (2007). Updated world map of the Köppen-Geiger climate classification. Hydrology and Earth System Sciences, 11, 1633-1644.

Rivas, I., Güitrón, A., \& Montero, M. (2011). Hydrologic vulnerability to climate change of the Lerma-Chapala Basin, Mexico. Sustainability Today, 297-308.

Robles-Morua, A., Che, D., Mayer, A. S., \& Vivoni, E. R. (2015). Hydrological assessment of proposed reservoirs in the Sonora River Basin, Mexico, under historical and future climate scenarios. Hydrological Sciences Journal, 60(1), 50-66.

Sedue, Secretaría de Desarrollo Urbano y Ecología. (1986). Estudio de calidad y clasificación del río Tampaón, San Luis Potosí y Querétaro México, DF: Secretaría de Desarrollo Urbano y Ecología.

Tapia, E. M., Minjarez, I., Espinoza, I., \& Minjarez, C. (2014). Use of Stella Software for the modelling of climate change impacts on water balance for the Rio Yaqui Basin, Sonora, Mexico. European Scientific Journal, ESJ, 10(14), 351-360.

Teutschbein, C., Wetterhall, F., \& Seibert, J. (2011). Evaluation of different downscaling techniques for hydrological climate-change impact studies at the catchment scale. Climate Dynamics, 37, 2087-2105. 
Troin, M., Velázquez, J. A., Caya, D., \& Brissette, F. (2015). Comparing statistical post-processing of regional and global climate scenarios for hydrological impacts assessment: A case study of two Canadian catchments. Journal of Hydrology, 520, 268-288.

Velázquez, J. A., Schmid, J., Ricard, S., Muerth, M. J., Gauvin St-Denis, B., Minville, M., Chaumont, D., Caya, D., Ludwig R., \& Turcotte, R. (2013). An ensemble approach to assess hydrological models' contribution to uncertainties in the analysis of climate change impact on water resources. Hydrology and Earth System Sciences, 17, 565-578, DOI: 10.5194/hess-17-565-2013

Velázquez, J. A., Troin, M., \& Caya, D. (2015). Hydrological modeling of the Tampaon River in the context of climate change. Tecnología y ciencias del agua, 6(5), 17-30.

Velázquez-Zapata, J. A., Troin, M., \& Dávila-Ortiz, R. (2017). Evaluación del impacto del cambio climático en los indicadores hidrológicos de una cuenca del centro de México con base en un ensamble de modelos climáticos y en el modelo hidrológico SWAT. Ingeniería, Investigación y Tecnología, 18(3), 341-351. 